\title{
Subjective adequacy of dialysis; a neglected concept in hemodialysis adequacy
}

\author{
Vajihe Biniaz ${ }^{1}$, Hossein Karimi Moonaghi2 ${ }^{*}$, Razieh Froutan ${ }^{1}$, Abbass Ebadi ${ }^{3}$ \\ ${ }^{1}$ Department of Medical Surgical Nursing, School of Nursing and Midwifery, Mashhad University of Medical Sciences, Mashhad, Iran \\ ${ }^{2}$ Evidence-Based Caring Research Center, Department of Medical Surgical Nursing, School of Nursing and Midwifery, \& Department of Medical \\ Education, School of Medicine, Mashhad University of Medical Sciences, Mashhad, Iran \\ ${ }^{3}$ Behavioral Sciences Research Center, Life style institute, Faculty of Nursing, Baqiyatallah University of Medical Sciences, Tehran, Iran
}

\section{A R T I C L E I N F O}

\section{Article Type:}

Original

\section{Article History:}

Received: 1 February 2018

Accepted: 4 May 2018

Published online: 13 May 2018

\section{Keywords:}

Hemodialysis

Dialysis adequacy

Content analysis

\begin{abstract}
A B S T R A C T
Introduction: Dialysis adequacy is a predictive factor for mortality and hemodialysis outcomes.

Objectives: Given the limited knowledge regarding dialysis adequacy, we sought to investigate the perceptions and experiences of patients as to subjective dialysis adequacy.

Materials and Methods: This qualitative study was carried out using the conventional content analysis method. We enrolled 25 hemodialysis patients, their companions, nurses, and nephrologists residing in Mashhad, Iran. We selected the participants through purposive sampling. To collect the data, we used semi-structured interviews and recording field notes until reaching data saturation. The data was analyzed by using Hsieh and Shannon qualitative content analysis approach.

Results: From the experiences of the patients regarding dialysis adequacy, five categories of inner wholeness, well-being, positive social interaction, effective self-empowerment and improvement of economic conditions were extracted.

Conclusion: The obtained results can play a key role in implementation of effective interventions for models of hemodialysis clinical function to lower the rate of mortality and promote quality of life among dialysis patients.
\end{abstract}

\section{Implication for health policy/practice/research/medical education}

In the present study, profound investigation of experiences of hemodialysis patients clarified the concept of subjective dialysis adequacy and determined its indices. The obtained results, along with expanding the nursing knowledge, pave the way for creating a specific tool for measuring the aspects of subjective dialysis adequacy in hemodialysis patients. This tool can play a key role in implementation of effective interventions in models of hemodialysis clinical function to lower the rate of mortality and promote quality of life among dialysis patients.

Please cite this paper as: Biniaz V, Karimi Moonaghi H, Froutan R, Ebadi A. Subjective adequacy of dialysis; a neglected concept in hemodialysis adequacy. J Renal Inj Prev. 2018;7(3):164-170. doi: 10.15171/jrip.2018.40.

\section{Introduction}

End-stage renal disease (ESRD) is a stage at which the patient requires renal replacement therapies, such as hemodialysis, peritoneal dialysis, or kidney transplant, for survival (1). Conceptual (2), cultural, educational, structural, and organizational barriers to peritoneal dialysis $(3,4)$ have made hemodialysis the most common life-sustaining treatment (5-8).

Neither the recent technical advancements in the production of monitoring tools nor improved quality of ultrafiltration machines and materials have been able to affect the consistently high mortality rate (15\%-25\%) in these patients and promote their quality of life.

Studies have ascribed that hemodialysis adequacy is a prominent predictor of mortality and hemodialysis outcomes (9). Patients undergoing hemodialysis with optimum quality have an equal longevity as those receiving renal transplant (10).

The multidimensional concept of dialysis adequacy (11) entails two interwoven aspects of objective and subjective (12). Based on former studies, adequate dialysis can affect short- and long-term morbidity and mortality of hemodialysis patients (13) and can result in subjective well-being (14). Twardowski in 2003 illustrated that 
adequate dialysis is hinged upon a set of subjective and objective criteria indicating treatment efficacy (15).

The objective aspect of dialysis is associated with indices such as Kt/V that are able to gauge the physiologic aspects of hemodialysis efficacy (16), whereas the subjective aspect of dialysis adequacy cannot be dependent on blood and fluids clearance of waste substances or calculation of a clinical parameter disregarding the idiosyncratic features of each individual.

This subjective aspect is associated with the experiences and perceptions of a patient regarding an adequate dialysis, his/her subjective perception of the physical and psychological signs, as well as feelings and expectations from the level of well-being during dialysis (17).

\section{Objectives}

Given the scarcity of studies and knowledge as to experiences of hemodialysis patients regarding dialysis adequacy and the limited perception of subjective dialysis adequacy, we sought to gain a profound understanding of subjective dialysis adequacy based on the experiences of hemodialysis patients.

\section{Patients and Methods}

\section{Study population}

In order to delineate the perceptions and experiences of hemodialysis patients regarding subjective dialysis adequacy, this qualitative study was designed and conducted using the conventional content analysis method during 2016-2017 (18).

Purposive sampling was performed from among hemodialysis patients who were willing to participate in the study (19). To have maximum diversity in demographic and phenomenological variables selective sampling was continued until reaching data saturation $(20,21)$. Faceto-face semi-structured interviews were conducted with 25 participants living in Mashhad (19 hemodialysis patients, 2 patient companions, 2 dialysis nurses, and 2 nephrologists). Additional demographic characteristics of the participants are shown in Table 1 .

The inclusion criteria were at least 12 weeks of regular hemodialysis (3-4 hours each session), age $>18$ years, basic education, no history of drug or alcohol abuse, and no history of hospital admission due to psychological problems during the last year.

The interviews were initiated with general questions such as 'describe your experiences in a hemodialysis day' and then continued with more specific queries such as 'what is your expectation of an adequate dialysis?' The participants were asked to state their experiences regarding hemodialysis adequacy. To gain more indepth information, exploratory questions were used. The interviews were performed during 40-110 minutes, and the setting was appointed with the agreement of the participants. Thereafter, the obtained data was analyzed by using Hsieh and Shannon's content analysis method (22). In this method, codes and categories were extracted from the raw materials inductively and systematically.

Table 1. Characteristics of participants

\begin{tabular}{|c|c|c|c|c|}
\hline Sex- Age & Hemodialysis vintage (month) & Education & Interview time & Participant Number \\
\hline F-49 & 171 & Primary or secondary & 53 & 1 \\
\hline F-73 & 40 & Primary or secondary & 41 & 2 \\
\hline $\mathrm{F}-42$ & 32 & Primary or secondary & 49 & 3 \\
\hline F-52 & 41 & College or university & 43 & 4 \\
\hline F-76 & 75 & Primary or secondary & 40 & 5 \\
\hline F-52 & - & College or university & 16 & 6 \\
\hline F-29 & - & College or university & 18 & 7 \\
\hline M-70 & 69 & College or university & 51 & 8 \\
\hline M-66 & 120 & College or university & 112 & 9 \\
\hline M-39 & 69 & College or university & 47 & 10 \\
\hline F-30 & 12 & College or university & 47 & 11 \\
\hline F-30 & 45 & College or university & 48 & 12 \\
\hline F-26 & 50 & College or university & 46 & 13 \\
\hline F-33 & 18 & College or university & 45 & 14 \\
\hline F-37 & 24 & College or university & 41 & 15 \\
\hline F-35 & 12 & College or university & 42 & 16 \\
\hline F-35 & 16 & College or university & 53 & 17 \\
\hline $\mathrm{F}-40$ & 192 & College or university & 61 & 18 \\
\hline$F-72$ & 40 & Primary or secondary & 40 & 19 \\
\hline$M-30$ & 72 & Primary or secondary & 67 & 20 \\
\hline M-37 & 75 & Primary or secondary & 75 & 21 \\
\hline M-33 & 75 & Primary or secondary & 63 & 22 \\
\hline$M-47$ & 264 & Primary or secondary & 92 & 23 \\
\hline M-56 & - & Primary or secondary & 33 & 24 \\
\hline$M-49$ & - & Primary or secondary & 16 & 25 \\
\hline
\end{tabular}


The regular presence of the researcher in different working shifts at the hemodialysis ward and her/his longtime involvement with the subject, as well as selection of the participants with maximum diversity (in terms of age, gender, occupational status, marital status, financial status, and history of hemodialysis) bolstered validity of the study. Member check and rectifying the codes that did not accurately describe the point of view of the participants (based on their own opinion) improved reliability of the study. To promote confirmability, the researcher first recorded her/his own presumptions regarding the answers and tried not to emphasize on them. Finally, dependability was assured by immediate transcription of the interviews and use of external check and reviewing the data. Further, with precise delineation of the study procedures and stages, the readers were able to follow the steps and appraise them.

\section{Ethical considerations}

The research followed the tenets of the Declaration of Helsinki. The observed ethical considerations in this study included obtaining approval from Ethics Committee of Mashhad University of Medical Sciences (code \# IR.MUMS.REC.1395.309), taking informed consent from the participants after explaining the objectives of the study, roles of researchers and participants, and the methods of data collection and recording, providing a quiet and peaceful environment during the interviews, giving the participants the right to withdraw from the study at any time, assuring confidentiality of data, and providing the results to the participants in case they wanted them.

\section{Results}

The mean age of the participants was $43.9 \pm 5.8$ years and their mean duration of hemodialysis was $71.8 \pm 65.1$ months. Sixty-one percent of the participants were female. Around 997 initial codes were extracted under five categories of inner wholeness, well-being, positive social interaction, effective self- empowerment and improvement of economic conditions. Each of these categories comprised of some sub-categories. Table 2 illustrates the process of shaping these categories, and subcategories.

\section{Inner wholeness}

Inner wholeness is an overwhelming feeling in hemodialysis patients, which makes them more tolerant and resilient in dealing with problems and stressful situations and managing them. The experience of internal wholeness in hemodialysis patients participating in this study was perceived as having emotional, mental, and spiritual well-being, as well as positive body image.

\section{Emotional health}

Following an adequate dialysis, the participants reported less restlessness, anxiety, and bafflement. In addition, they were not depressed, irritable, or dissociable and felt more
Table 2. Study categories and subcategories

\begin{tabular}{ll}
\hline Emerged categories & Subcategories \\
\hline \multirow{3}{*}{ Inner wholeness } & Emotional health \\
& $\begin{array}{l}\text { Mental health } \\
\text { Spiritual health } \\
\text { Positive body image }\end{array}$ \\
& Self-acceptance \\
& Understanding Satisfaction \\
Well being & Understanding the purpose \\
& Feeling good \\
& Understanding security \\
Positive social interactions & $\begin{array}{l}\text { Normal social function } \\
\text { Understanding support }\end{array}$ \\
Effective self-empowerment & Voluntary exploration of health literacy \\
& Foluntary adherence to treatment \\
Improving economic & Occupational prosperity \\
conditions & Reduced treatment costs \\
\hline
\end{tabular}

peaceful. Participant No. 1 in this regard said; 'When my dialysis is good, I feel happy and socialize with everyone, as people in Mashhad say 'I have a wedding in myself (laughing)'. I like to work out to stay that way. I go to all events whether wedding or mourning. Even when I want to go to a wedding, I receive an additional dialysis session to be happy and have a good time'

\section{Mental health}

The participants experienced mental health after a highquality dialysis; they had higher tolerance for irritating stimulants such as noise, unpleasant smells, heat, cold, and light.

They also felt relaxed and had excellent sleep. Maintaining a neat appearance, positive inculcation and tolerance of others were among the other experiences of the participants. Participant No. 13 in this respect asserted: "'After an optimal dialysis, I feel so relaxed, I can tolerate anything, I'm in the mood for my nieces and nephews, I do not shout at them to go away, I can stand the noise from TV and I do not argue with anyone."

\section{Spiritual health}

Spiritual well-being after a high-quality dialysis was perceived as experience of patience in the face of illness and considering sickness as a divine test in our participants. They also established a better spiritual connection with God and along with a sense of thankfulness, relied on him and were satisfied with his satisfaction. In this respect, participant No. 21 confirmed; "believing in God and the fact that God does not leave us alone was the most important factor that I could get along with dialysis, and my trust in God has made me feel satisfied with my life."

\section{Positive body image}

The hemodialysis patients reported positive body image after a high-quality dialysis. Positive self-image entails an emphasis on strengths rather than physical flaws, focus on 
skills and fulfillments, and positive attitude towards one's appearance and the new body shape with the catheters. Our participants did not compare their own appearance with normal people and were not willing to limit their social relationships because of the changes in their appearance or others' opinion about them. Participant No. 1 expressed 'When I look at the mirror, I don't think I look strange. Nothing is wrong with me and there is no reason for others to look at me. Until I roll up my sleeves no one understands I receive dialysis. My appearance is so normal that I came from work for dialysis and went back there for 10 years without anyone understanding it;

\section{Well-being}

The sense of well-being in hemodialysis patients is a cognitive feeling that stems from the pleasant feeling of being well. This feeling comes along with a positive attitude toward the surrounding world and the pleasure of being alive. From the analysis of this category, selfacceptance, perception of satisfaction, sense of well-being, understanding of purpose, and understanding of security were inferred.

\section{Self-acceptance}

Participants in this study, after an adequate dialysis, felt better with accepting their new condition. Participant No. 18 said; "My condition was very different before the illness, but this is the new condition and I have accepted it. I have settled with my illness, so it doesn't annoy me."

\section{Understanding satisfaction}

A hemodialysis patient, experiencing a sense of wellbeing, is satisfied with his/her life and feels passionate interest about life. Participant No. 21 expressed: "one must be satisfied in every stage of life. I have God, I have my wife and we love each other. She is a healthy and patient woman and helps me a lot. I have healthy kids, who love me so much. I can work hard and make the ends meet. I'm happy."

\section{Feeling good}

Participants in this study experienced a sense of wellbeing after an optimal dialysis that was accompanied with a sense of vitality and happiness. Participant No. 1 asserted: "I don't consider myself as ill. I come here and go on my own feet and I live my life and do my job. On the contrary, I feel that now I appreciate my life more. I feel happy inside."

\section{Understanding the purpose}

The experience of a sense of well-being by the participants was accompanied by a sense of success and achievement. Participant No. 21, who after 75 months of dialysis, still continues to work as a builder, says: "when I think, I find myself as a successful person because with this disease and its problems I still can make ends meet. I could resolve all my problems and get all the things I aspired. When I think of these things, I feel very good and relaxed."

\section{Understanding security}

The sense of security was another experience of the hemodialysis patients in this study. Participant No. 21 said; "Now I feel more secure. First of all, I rely on God and then my family and children. I have no concern for future, I'm so assured.

\section{Positive social interactions}

After an adequate dialysis, the hemodialysis patients participating in this study had a high inclination for interaction and had social communication with others, which included a positive social interaction with family members, relatives, acquaintances, friends, colleagues, or doctors, nurses, caregivers and other patients in the dialysis ward. These interactions caused the hemodialysis patients to gain support from others. From the analysis of this category, normal social function and perceived support were inferred.

\section{Normal social function}

The participants also mentioned improved social relationship. Following an adequate dialysis, they felt more power over their life and were more determined for decision-making. On this subject, participant No. 23 who was a factory manager said; 'When I have a good dialysis, I feel so different that everyone at home becomes surprised that I'm smiling. As if I choose a better way for my life. When I feel well, I manage everything better. My motivation is higher and I'm more determined for a good life'.

\section{Understanding support}

Participants, using positive social interactions, could benefit from experiences of other dialysis patients and attract the support of their family and healthcare team. Participant No. 22 stated; "My family didn't know what dialysis was. They thought my blood needs to be filtered and only my dialysis friends and nurses and doctors had my back and could help me a great deal."

\section{Effective self-empowerment}

Effective self-empowerment is a voluntary, active, and purposeful behavior in hemodialysis patients, who are motivated to protect their mental and physical health, meet social and psychological needs, or prevent accidents and illnesses. Effective self-empowerment includes voluntary exploration of health literacy, voluntary adherence to treatment, and focus on self-care behaviors.

\section{Voluntary exploration of health literacy}

After having optimal dialysis and due to the good feeling following that, the participating hemodialysis patients were interested in finding information that sustained this pleasant mood and sought to answer health-related questions from any valid or invalid source. Participant 
No. 15 said; "I have read the educational pamphlets in the ward for the complications of dialysis. Sometimes I use the Internet to answer my questions."

\section{Voluntary adherence to treatment}

A behavior is deliberate and purposeful when the patient matches his or her behaviors with treatment recommendations provided by health care providers such as a doctor or nurse. Participant No. 9 asserted; "When I have a good dialysis, I take more care of myself and the fistula, I'm careful about what I eat, I try to do whatever my doctor has recommended".

\section{Focus on self-care behaviors}

These behaviors are focused on taking medications, adhering to diet, changing lifestyle, or observing continuity in clinical referrals. Participant No. 9 responded; "I don't go to overcrowded places to avoid catching a cold, flu, or other diseases. I'm so careful about food's salt and oil and I meticulously pick fruits and snacks. I disinfect the house with Espand."

\section{Improving economic conditions}

The economic conditions in a hemodialysis patient's life after an adequate dialysis change in such a way that family income increases at least as much as the dialysis expenses. The improvement achieved in the economic context can be the result of a greater chance of finding a job with better conditions or reducing the disease-related costs. The upgrowth in the economic status involves occupational prosperity and reduced treatment costs.

\section{Occupational prosperity}

The participating hemodialysis patients, after performing high-quality dialysis, had better job conditions due to better physical status, increased strength and energy for work, and changes in their psychological conditions, and consequently, they had better economic conditions. Participant No. 22 claimed; "My boss has promoted my payment. He's more satisfied with my work because my physical power is relatively higher. I can work more easily."

\section{Reduced treatment costs}

Participants experienced a better economic situation after an optimal dialysis due to reduced hospital admissions or fewer doctor visits or less need for expensive drugs. Participant No. 7 expressed; "Well, obviously, when dialysis is adequate the medication and physician expenses are lower, as well."

\section{Discussion}

The findings of this study showed that hemodialysis patients, after optimal dialysis, have pleasant nonphysiological experiences indicating the psychological, social, educational, and economic concepts of hemodialysis adequacy.

Gregory et al, in a qualitative study on ESRD patients showed that these patients shaped a new identity for themselves, which is constantly changing and is the result of perception of the patients regarding their vague future, the disease-related needs, and constant dependence on dialysis machine, drugs, and care providers (23). Likewise, in this study, we noted that patients with adequate dialysis accept a new self that is adapted to the new conditions; this acceptance produces a sense of well-being in them. Kimmel et al pinpointed that disease perception in hemodialysis patients was associated with lower probability of survival and adherence to treatment (24). Additionally Mittal et al confirmed that hemodialysis patients, compared to those inflicted with other chronic diseases, find their physical health significantly reduced (25). Chilcot et al in a study on hemodialysis patients illustrated that disease perception is an important predictor of disease adaptation (26). Similarly, individuals benefiting from adequate dialysis accepted dialysis and its limitations in the form of self-empowerment.

Former studies proposed that outcome of an adequate dialysis is the sense of subjective well-being (27). Our results also indicated that hemodialysis patients after a high-quality dialysis feel healthy, well, happy, safe, successful, and comfortable, which were entitled sense of well-being.

The performed studies on quality of life illustrated the prominent effects of age on the physical aspect of quality of life. HEMO study with a large cohort of patients demonstrated the elderly patients perceived the reduced quality of life less than younger ones (higher quality of life among elderly patients), and compensated for the deteriorated physical status with spiritual aspects (28). This finding confirmed our results. In the current study, elderly hemodialysis patients (older than 65 years) experienced higher life satisfaction than younger ones, which could be due to the fact that the limitations caused by the treatment disrupted younger patients' life to a larger extent.

Approximately all the dialysis patients had worries regarding their sexual health after treatment, which affected their quality of life (29). Based on the evidence, about half of the 20-50 years old men were suffering from erectile problems (30). Strippoli et al indicated that $84 \%$ of hemodialysis patients were suffering from sexual problems (31). Furthermore, Kim et al showed a significant reduction in all aspects of sexual relationship (i.e., desire, arousal, lubrication, orgasm, and pain) in hemodialysis women. Song et al (32) and Soykan et al (33) also presented that dialysis adequacy (Kt/V higher than 1.3) had no significant effect on improving sexual disorders. In our study, hemodialysis patients after having a high-quality dialysis experienced more satisfaction with their marital relationship which created a sense of wellbeing.

Depression was one of the most prevalent disorders in hemodialysis patients (34). There were some reports on better quality of life and diminished depression with enhanced spiritual perception (35). Some other studies 
indicated the positive effects of spirituality on the rate of mortality and sense of well-being (36-38). Likewise, in our study, spiritual well-being arising from the perception of spiritual experiences, such as trust in God and praying for healing, led to the feeling of inner wholeness.

Kabahizi et al ascribed that dialysis adequacy had a significant positive relationship with physical wellbeing and higher physical function, such that high dialysis adequacy resulted in high-quality physical role and significantly decreased role limitations due to lack of physical health (39). In congruence, we found that quality of dialysis enhances occupational prosperity and economic conditions by improving physical condition and increasing the individual's energy and power to carry out tasks.

\section{Conclusion}

In the present study, profound investigation of experiences of hemodialysis patients clarified the concept of subjective dialysis adequacy and determined its indices. The obtained results, along with expanding the nursing knowledge, pave the way for creating a specific tool for measuring the aspects of subjective dialysis adequacy in hemodialysis patients. This tool can play a key role in implementation of effective interventions in models of hemodialysis clinical function to lower the rate of mortality and promote quality of life among dialysis patients.

\section{Acknowledgments}

This article is part of a Ph.D. thesis with code 941689 that was approved by Mashhad University of Medical Sciences. The authors sincerely thank all the participants in this research.

\section{Conflicts of interest}

The authors declare no conflicting interest.

\section{Authors' contribution}

VB developed the original idea and the protocol, abstracted and analyzed data, wrote the manuscript. HKM, RF and AE contributed to the development of the protocol, abstracted data, and prepared the manuscript.

\section{Ethical considerations}

Ethical issues (including plagiarism, data fabrication, double publication) have been completely observed by the authors.

\section{Funding/Support}

This research was financially supported by Mashhad University of Medical Sciences.

\section{References}

1. Grassmann A, Gioberge S, Moeller S, Brown G. ESRD patients in 2004: global overview of patient numbers, treatment modalities and associated trends. Nephrol Dial Transplant. 2005;20:2587-93. doi: 10.1093/ndt/gfi159.
2. Desmet JM, Fernandes V, des Grottes JM, Spinogatti N, Collart F, Pochet JM, et al. Perceptive barriers to peritoneal dialysis implementation: an opinion poll among the Frenchspeaking Belgian nephrologists. Clin Kidney J. 2013;6:35862. doi: $10.1093 / \mathrm{ckj} / \mathrm{sft} 041$.

3. Termorshuizen F, Korevaar JC, Dekker FW, Van manen JG, Boeschoten EW, Kredit RT. Hemodialysis and peritoneal dialysis: comparison of adjusted mortality rates according to the duration of dialysis: analysis of The Netherlands Cooperative Study on the adequacy of dialysis 2. J Am Soc Nephrol. 2003;14:2851-60. doi: 10.1097/01. ASN.0000091585.45723.9E.

4. Troidle L, Kliger A, Finkelstein F. Barriers to utilization of chronic peritoneal dialysis in network \#1, New England. Perit Dial Int. 2006;26:452-7.

5. Beekmann SE, Henderson DK. Infections caused by percutaneous intravascular devices. In: Mandell GL, Bennett JE, Dolin R, eds. Principles and practice of infectious Disease. 7th ed. Churchill Livingstone Elsevier; 2010.p.3697-716.

6. Smeltzer SC, Bare BG, Hinkle JL, Cheever KH. Brunner and Suddarth's Textbook of Medical-Surgical Nursing. 12th ed. Philadelphia: JB Lippincott Co; 2010.

7. Sehgal AR, Leon JB, Siminoff LA, Singer ME, Bunosky LM, Cebul RD. Improving the quality of hemodialysis treatment: a community-based randomized controlled trial to overcome patient-specific barriers. JAMA 2002;287:19617. doi: 10.1001/jama.287.15.1961

8. Juergensen E, Wuerth D, Finkelstein SH, Juergensen PH, Bekui A, Finkelstein F. Hemodialysis and peritoneal dialysis: patients' assessment of their satisfaction with therapy and the impact of the therapy on their lives. Clin J Am Soc Nephrol 2006;1:1191-6. doi: 10.2215/CJN.01220406

9. Abdelwahab H, Shigidi M, El-Tohami A, Ibrahim L. Adherence of healthcare professionals to evidencebased clinical practice guidelines in the management of hemodialysis patients, Khartoum State, Sudan. Arab J Nephrol Transplant 2013;6:99-104.

10. Saad MM, El Douaihy Y, Boumitri C, Rondla C, Moussaly E, Daoud M, El Sayegh SE. Predictors of quality of life in patients with end-stage renal disease on hemodialysis. Int J Nephrol Renovasc Dis. 2015;8:119-23. doi: 10.2147/IJNRD. S84929.

11. Kerr P, Perkovic V, Petrie J, Agar J, Disney A; Caring for Australians with Renal Impairment (CARI). The CARI guildlines. Dialysis adequacy (HD) guidelines. Nephrology (Carlton). 2005;10:S61-80. doi: 10.1111/j.14401797.2005.00464_1.x

12. Fouad T, Mokhtar Y. Principle factors affecting adequacy of dialysis. Assessment of adequacy of dialysis. Doctors lounge; 2003. http://www.doctorslounge.com/nephrology/articles/ dialysis_adequacy/adeq1.htm.

13. Canaud B. Adequacy target in hemodialysis. J Nephrol. 2004; 17:S77-86.

14. Kahneman D, Krueger AB. Developments in the measurement of subjective well-being. J Econ Perspect. 2006;20:3-24. doi: 10.1257/089533006776526030

15. Twardowski ZJ. We should strive for optimal hemodialysis: A criticism of the hemodialysis adequacy concept. Hemodial Int 2003; 7: 5-16. doi: 10.1046/j.1492-7535.2003.00002.x.

16. Davenport A. Differences in prescribed Kt/V and delivered haemodialysis dose-why obesity makes a difference to survival for haemodialysis patients when using a 'one size fits all' Kt/V target. Nephrol Dial Transplant. 2013;28:iv219-23. doi: $10.1093 / \mathrm{ndt} / \mathrm{gft} 237$. 
17. Al Onazi M, Al Jondeby M, Azeem M, Al Sayyari A. Factors affecting Saudi hemodialysis patients' perception of healthcare providers' empathy. Arab J Nephrol Transplant. 2011;4:71-6.

18. Forman J, Damschroder L. Qualitative content analysis. In: Jacoby L, Siminoff LA, eds. Empirical Methods for Bioethics: A Primer (Advances in Bioethics, Volume 11). Emerald Group Publishing Limited; 2007. p. 39-62.

19. Coyne IT. Sampling in qualitative research. Purposeful and theoretical sampling; merging or clear boundaries? J Adv Nurs. 1997;26:623-30. doi: 10.1046/j.1365-2648.1997.t01-2500999.x

20. Mayring P. Qualitative content analysis: theoretical foundation, basic procedures and software solution. Klagenfurt: SSOAR; 2014. http://nbn-resolving.de/ urn:nbn:de:0168-ssoar-395173.

21. Cleary M, Horsfall J, Hayter M. Data collection and sampling in qualitative research:does size matter? J Adv Nurs. 2014;70(3):473-5

22. Hsieh HF, Shannon SE. Three approaches to qualitative content analysis. Qual Health Res. 2005;15:1277-88. doi: $10.1177 / 1049732305276687$

23. Gregory DM, Way CY, Hutchinson TA, Barrett BJ, Parfrey PS. Patients' perceptions of their experiences with ESRD and hemodialysis treatment. Qual Health Res. 1998;8:764-83. doi: 10.1177/104973239800800604

24. Kimmel PL, Peterson RA, Weihs KL, Simmens SJ, Alleyne $\mathrm{S}$, Cruz L, et al. Psychosocial factors, behavioral compliance and survival in urban hemodialysis patients. Kidney Int. 1998;54:245-54. doi: 10.1046/j.1523-1755.1998.00989.x

25. Mittal SK, Ahern L, Flaster E, Maesaka JK, Fishbane S. Selfassessed physical and mental function of haemodialysis patients. Nephrol Dial Transplant. 2001;16;1387-94. doi: 10.1093/ndt/16.7.1387

26. Chilcot J, Wellsted D, Farrington K. Illness perceptions predict survival in haemodialysis patients. Am J Nephrol. 2011;33:358-63. doi: 10.1159/000326752.

27. Santos PR. Subjective well-being measures of hemodialysis patients. In: Penido MG, ed. Technical Problems in Patients on Hemodialysis. InTech; 2011.

28. Rocco MV, Cheung AK, Greene T, Eknoyan G. The HEMO Study: applicability and generalizability. Nephrol Dial Transplant. 2005;20:278-84. doi: 10.1093/ndt/gfh304
29. Peng YS, Chiang CK, Kao TW, Hung KY, Lu CS, Chiang SS, et al. Sexual dysfunction in female hemodialysis patients: a multicenter study. Kidney Int. 2005;68:760-5. doi: 10.1111/j.1523-1755.2005.00454.x

30. Yilmaz M, Özaltın G. The sexual behaviors of patients receiving peritoneal dialysis treatment. Sex Disabil. 2011;29:21-32. doi: 10.1007/s11195-011-9199-3

31. Strippoli GF, Sexual Dysfunction (CDS) in Hemodialysis Working Group. Sexual dysfunction in women with ESRD requiring hemodialysis. Clin J Am Soc Nephrol. 2012; 7:97481. doi: $10.2215 /$ CJN.12601211

32. Song YS, Yang HJ, Song ES, Han DC, Moon C, Ku JH. Sexual function and quality of life in Korean women with chronic renal failure on hemodialysis: case-control study. Urology. 2008;71:243-6. doi: 10.1016/j.urology.2007.10.020.

33. Soykan A, Boztas H, Kutlay S, Ince E, Nergizoglu G, Dilekoz AY, Berksun O: Do sexual dysfunctions get better during dialysis? Results of a six-month prospective follow-up study from Turkey. Int J Impot Res. 2005;17:359-63. doi: 10.1038/ sj.ijir.3901324

34. Cukor D, Peterson RA, Cohen SD, Kimmel PL. Depression in end-stage renal disease hemodialysis patients. Nat Clin Pract Nephrol.2006;2:678-87. doi: 10.1038/ncpneph0359

35. Bennett PN, Weinberg MK, Bridgman T, Cummins RA. The happiness and subjective well-being of people on haemodialysis. J Ren Care. 2015;41:156-61. doi: 10.1111/ jorc. 12116 .

36. Martinez BB, Custodio RP. Relationship between mental health and spiritual wellbeing among hemodialysis patients: a correlation study. Sao Paulo Med J. 2014;132:23-7. doi: 10.1590/1516-3180.2014.1321606.

37. Griva K, Jayasena D, Davenport A, Harrison M, Newman SP. Illness and treatment cognitions and health related quality of life in end stage renal disease. Br J Health Psychol. 2009;14:17-34. doi: 10.1348/135910708X292355.

38. Eslami AA, Rabiei L, Khayri F, Nooshabadi MR, Masoudi R. Sleep quality and spiritual well-being in hemodialysis patients. Iran Red Crescent Med J. 2014;16:e17155. doi: 10.5812/ircmj.17155

39. Kabahizi J. Impact of Dialysis Adequacy on Patient Outcomes (dissertation). Johannesburg: University of the Witwatersrand; 2006.

Copyright $(2018$ The Author(s); Published by Nickan Research Institute. This is an open-access article distributed under the terms of the Creative Commons Attribution License (http://creativecommons.org/licenses/by/4.0), which permits unrestricted use, distribution, and reproduction in any medium, provided the original work is properly cited. 